\title{
Der Einfluss des F-18-FDG-PET/CT auf das stadien- bezogene Gesamtüberleben beim fortgeschrittenen nicht-kleinzelligen Lungenkarzinom (NSCLC) ${ }^{*}$
}

\author{
Staging with ${ }^{18} \mathrm{~F}-\mathrm{FDG}-\mathrm{PET} / \mathrm{CT}$ Influences Stage-Specific Survival in Advanced \\ Non-Small Cell Lung Cancer (NSCLC)
}

Autoren

Institute
S. Tönnies ${ }^{1}$, T. T. Bauer ${ }^{1}$, D. Misch' ${ }^{1}$, C. Boch ${ }^{1}$, T. Blum¹ , R. C. Bittner ${ }^{2}$, G. J. Förster ${ }^{3}$, J. Kollmeier ${ }^{1}$

'Lungenklinik Heckeshorn, Klinik für Pneumologie, HELIOS Klinikum Emil von Behring, Berlin

${ }^{2}$ Institut für Diagnostische und Interventionelle Radiologie, HELIOS Klinikum Emil von Behring, Berlin

${ }^{3}$ Institut für Nuklearmedizin und molekulare Bildgebung, HELIOS Klinikum Emil von Behring, Berlin

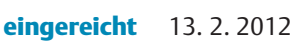
akzeptiert nach Revision 12. 3.2012

Bibliografie DOI http://dx.doi.org/ $10.1055 / s-0032-1308916$ Pneumologie 2012; 66: 212-217 (c) Georg Thieme Verlag KG Stuttgart · New York ISSN 0934-8387

\section{Korrespondenzadresse} Dr. med. Jens Kollmeier Lungenklinik Heckeshorn Klinik für Pneumologie HELIOS Klinikum Emil von Behring Walterhöferstr. 11 14164 Berlin jens.kollmeier@helioskliniken.de

\section{Zusammenfassung \\ $\nabla$}

Hintergrund: Das PET/CT mit F-18-Fluordesoxyglukose (FDG) hat eine hohe Sensitivität (89$100 \%$ ) und zufriedenstellende Spezifität(79-95\%) für die Diagnose des NSCLC. Gegenwärtig wird es überwiegend im präoperativen Staging eingesetzt. Dies führt in ca. 15\% aller Fälle zu einer Diagnose von Fernmetastasen, die weder klinisch vermutet noch durch eine vorausgegangene konventionelle Bildgebung erkannt wurden. Wir prüften, ob die daraus resultierende Umstadiierung dieser Fälle einen Einfluss auf das statistische Gesamtüberleben von Patienten im palliativen Stadium IV haben kann.

Ziel: Vergleich des Gesamtüberlebens von Patienten mit Stadium IV NSCLC, die mittels FDG-PET/ CT stadiiert wurden, mit Patienten, bei denen ausschließlich konventionelle Bildtechnologien zum Stadiieren verwendet wurden.

Methoden: Wir haben das Gesamtüberleben aller Patienten unserer Klinik mit der Neudiagnose eines NSCLC im Stadium IV aus dem Jahre 2009 ( $n=254)$ analysiert. 96/254 (38\%) Patienten wurden mittels FDG-PET/CT stadiiert, 158/254 (62\%) mittels konventioneller Methoden (CT-Gruppe). Die Überlebensdaten wurden mittels KaplanMeier-Statistik verglichen.

Ergebnisse: Die Patienten in der PET/CT-Gruppe waren jünger $(65 \pm 11)$ als die der CT-Gruppe (68 \pm 10 Jahre, $p=0,008)$. Das mediane Gesamtüberleben aller Patienten lag bei 246 Tagen (Bereich: 217-275 Tage); 338 Tage für die PET/CTGruppe (Bereich: 247-429 Tage) und 207 für die CT-Gruppe (Bereich: 161-253 Tage, $p=0,001$ ), entsprechend einem Unterschied im medianen Gesamtüberleben von 131 Tagen (4,4 Monate).

Schlussfolgerung: Der heutige Einsatz des FDGPET/CT im Rahmen des überwiegend präoperativen Stagings beim NSCLC identifiziert eine prognostisch günstigere Gruppe an fernmetastasierten Patienten. Deren konsequente Umstadiierung

\section{Abstract \\ $\nabla$}

Background: PET/CT investigation with ${ }^{18} \mathrm{~F}-$ fluorodeoxyglucose (FDG) has a high sensitivity (89$100 \%$ ) and good specificity (79-95\%) for the diagnosis of NSCLC. Currently, it is mainly used in preoperative staging. This leads in approximately $15 \%$ of these cases to the diagnosis of metastatic disease that was neither clinically suspected nor seen in previously performed conventional imaging. We hypothesised that including these cases in the palliative stage IV group would have an influence on overall survival.

Aim: The aim of this study was to compare the overall survival (OS) of patients with stage IV NSCLC who underwent FDG-PET/CT staging with patients in whom conventional imaging procedures were performed.

Methods: We analysed the OS of all stage IV NSCLC patients diagnosed in our clinic in 2009 (n $=254$ ), 96/254 (38\%) patients were staged with PET/CT and 158/254 (62\%) with conventional imaging (CT group). Survival data were compared by Kaplan-Meier statistics.

Results: Patients in the PET/CT group were younger $(65 \pm 11)$ than in the CT group $(68 \pm 10$ years; $p=0.008)$. The median OS of all patients was 246 (range: 217 - 275) days; 338 (range: 247-429) days in the PET/CT group and 207 (range: $161-$ 253 ) days in the CT group $(p=0.001)$, stating a difference of 131 days ( 4.4 months) in median OS. Conclusion: The use of FDG-PET/CT staging mainly in the preoperative setting leads to stage migration of patients with a better prognosis into the worst stage (IV) and thus longer survival within this subgroup. This survival benefit is unrelated to treatment and needs to be addressed in future studies.

\footnotetext{
* Diese Studie wurde durch ein Stipendium der OskarHelene-Heim-Stiftung gefördert.
} 
in ein palliatives Stadium IV führt zu einer therapieunabhängigen Verlängerung des mittleren Gesamtüberlebens innerhalb dieses Stadiums.

\section{Einleitung}

$\nabla$

Das Lungenkarzinom ist weltweit die häufigste Form von Krebs und mit 40000 Sterbefällen pro Jahr die vierthäufigste Todesursache in der Bundesrepublik Deutschland [1]. Trotz neuer Therapieansätze hat sich dessen Prognose in den letzten Jahren nur geringfügig verbessert. Bis auf eine kleine Subgruppe von Patienten, bei denen spezifische Mutationen im Tumor nachgewiesen werden können, hat sich das mediane Überleben der Patienten mit einem primär metastasierten nicht-kleinzelligen Lungenkarzinom (NSCLC) im Stadium IV in den letzten 30 Jahren nur um wenige Monate verlängern lassen und liegt immer noch deutlich unter einem Jahr. 2007 hat die International Association for the Study of Lung Cancer (IALSC) zuletzt stadienbezogene Überlebenszeiten veröffentlicht, die für Patienten mit einem Stadium IV im Median sechs Monate betrugen [2]. Neben den therapeutischen Fortschritten haben sich in den letzten Jahren aber auch die diagnostischen Möglichkeiten weiterentwickelt. Bei der Diagnostik des Lungenkarzinoms kommen zur Stadieneinteilung neben invasiven Methoden traditionelle Bildtechnologien wie die Sonografie, die Computertomografie (CT), die Magnet-Resonanztomografie (MRT) sowie die Knochenszintigrafie zum Einsatz. Darüber hinaus findet man an vielen Kliniken mittlerweile Hybrid-Scanner, die die Positronen-Emissions-Tomografie (PET) mit der CT verbinden, das sogenannte PET/CT. Durch die zusätzliche Integrierung der PET zum CT werden nicht nur anatomischstrukturelle Information über das Gewebe abgebildet, sondern auch metabolische und biochemische Prozesse visualisiert, die durch exakte Überlagerung der Bilddaten den anatomischen Strukturen direkt zugeordnet werden können. Die meisten Tumorzellen zeichnen sich vor allem durch einen erhöhten Glukosestoffwechsel aus, der mithilfe von radioaktiv markiertem Zucker detektiert werden kann. Verwendet wird mit dem Positronenstrahler F-18 markierte Fluordesoxyglukose (F-18-FDG), die von den Zellen wie Glukose aufgenommen und phosphoryliert wird, dann aber in der Glykolyse nicht weiter verarbeitet werden kann und sich in den Zellen ansammelt. Mit dem PET/ CT-Scanner lassen sich Akkumulationen des FDG im Körper sehr genau detektieren, den anatomischen Strukturen zuordnen und sogar semiquantitativ messen. Beim nicht-kleinzelligen Lungenkarzinom lässt sich mit dieser diagnostischen Methode eine hohe Sensitivität (89-100\%) sowie Spezifität (78-95\%) erreichen [3]. Durch die Verbindung von physiologischen und anatomischen Informationen ist eine genauere Lokalisierung des Tumorgewebes möglich [4,5], zudem werden in $12-15 \%$ aller Fälle durch das PET/CT Fernmetastasen detektiert, die durch konventionelle Diagnostikmethoden ansonsten unerkannt bleiben würden [6, 7]. Folgerichtig empfiehlt die interdisziplinäre S3-Leitlinie der Deutschen Gesellschaft für Pneumologie und Beatmungsmedizin und der Deutschen Krebsgesellschaft das FDG-PET/CT zum Lymphknotenstaging und zum Ausschluss von Fernmetastasen für das klinische Stadium IB - IIIB (Empfehlungsgrad A) [8]. Demzufolge wird das PET/CT in Deutschland überwiegend in der präoperativen Diagnostik eingesetzt. Bei nahezu jedem fünften Patienten (18\% der Fälle) führt das PET zu einer Änderung des therapeutischen Prozedere [7]. So konnte unter anderem gezeigt werden, dass eine Stadieneinteilung mittels PET/CT bei Patienten mit nicht-kleinzelligem Lungenkarzinom zu einer Reduktion unnötiger Thorakotomien führt $[9,10]$ und dass diese Methode möglicherweise eine genauere Überwachung des Therapieerfolgs ermöglicht [11].

Wenn jedoch in $15 \%$ der Fälle durch das PET/CT ein Höherstufen in der Stadieneinteilung, ein sogenanntes „Upstaging“ erfolgt, so kommt es in dessen Folge auch zu Veränderungen der Patientencharakteristika innerhalb der einzelnen Stadien. 1985 beschrieben Feinstein et al. [12], dass die Migration prognostisch günstigerer Patienten mit NSCLC in ein schlechteres Stadium, bedingt durch eine modernere Diagnostik, in allen Stadien zu einem verlängerten Durchschnittsüberleben führte, einem sogenannten Will-Rogers-Phänomen entsprechend. Verglichen wurden damals die diagnostischen Möglichkeiten bzw. bildgebenden Verfahren von 1977 mit einem historischen Kollektiv aus den Jahren 1953 - 1964. Ob der heutige Einsatz des PET/CT auch Auswirkungen auf das stadienbezogene Überleben hat, ist bisher unklar. Mit dieser Studie wollten wir feststellen, ob der Einsatz eines PET/CT im Primärstaging beim NSCLC zu einer Verlängerung des Gesamtüberlebens im Stadium IV führen kann und abschätzen, wie groß der zu erwartende Effekt für prospektive Studien sein wird.

\section{Material und Methoden}

$\nabla$

\section{Patientenselektion}

Aus der Tumordatenbank der Lungenklinik Heckeshorn im HELIOS Klinikum Emil von Behring wurden alle Patienten mit der Neudiagnose eines nicht-kleinzelligen Lungenkarzinoms aus dem Jahr 2009 ausgewählt, um eine aussagefähige Nachbeobachtungszeit zu gewährleisten. Zu dieser Zeit wurde das FDG-PET/CT aufgrund der Indikations-Zulassung durch den Gemeinsamen Bundesausschuss (G-BA) überwiegend im Rahmen der präoperativen Diagnostik eingesetzt. Bei den so ermittelten 595 Patienten lag bei 254 (42\%) bereits primär ein Stadium IV vor. Es handelte sich um 104 (41\%) Frauen und 150 (59\%) Männer zwischen 37 und 90 Jahren.

Durch Auswertung der Bilddatenbank unseres Hauses (PACS) wurde retrospektiv festgestellt, dass 96/254 Patienten (38\%) primär ein FDG-PET/CT erhalten hatten und dieses zur Stadieneinteilung herangezogen wurde. Bei 158/254 Patienten (62\%) lag kein primäres FDG-PET/CT vor, die initiale Stadieneinteilung erfolgte somit mit konventioneller Bildgebung wie CT, MRT, Knochenszintigrafie und Sonografie. Ein Thorax-CT war bei allen Patienten durchgeführt worden. Nur Patienten, die zum primären Staging ein FDG-PET/CT erhielten, wurden in die PET/CT-Gruppe aufgenommen, alle für ein Re-Staging angefertigten FDG-PET/CT wurden nicht gewertet. Alle diagnostischen Untersuchungen wurden nach den allgemeinen diagnostischen Standards angefertigt.

Die Therapie der Patienten richtete sich selbstverständlich nicht nach den eingesetzten diagnostischen Mitteln. Als Erstlinientherapie wurde in aller Regel eine platinhaltige Kombinationschemotherapie eingesetzt.

Mit dem Stichtag 7.12.2010 wurden die Überlebensdaten aller untersuchten Patienten mit dem Einwohnermeldeamt Berlin abgeglichen.

\section{Statistik}

Es wurden zunächst Häufigkeiten für die Stadium-IV-Gruppe ermittelt und mittels Chi-Quadrat-Test verglichen. Mittelwerte wurden mit dem Student's T-Test verglichen und mit der Stan- 
dardabweichung dargestellt. Die Überlebenszeiten sind als Median \pm Spannweite oder als Mittelwerte mit den 95\% Konfidenzintervallen angegeben. Die Überlebensdaten wurden nach der Kaplan-Meier-Methode berechnet und in kumulativen Überlebenskurven dargestellt (Kaplan-Meier-Kurve). Diese Überlebenskurven wurden in der univariaten Statistik mittels Log-rank-Test verglichen. Für alle Statistiken wurde ein Signifikanzniveau von $5 \%$ angenommen. Alle Berechnungen wurden mittels SPSS (Vers. 15.0) durchgeführt.

\section{Ergebnisse}

Die Protokollgruppe umfasste insgesamt 254 Patienten mit nichtkleinzelligem Lungenkarzinom im Stadium IV. 96 Patienten wurden mittels PET/CT stadiiert, 158 Patienten durch konventionelle Bildgebung wie CT, MRT, Knochenszintigrafie und Sonografie. In der PET/CT-Gruppe waren 29 Patienten weiblich (30\%) und 67 männlich (70\%), in der CT-Gruppe verteilten sich die Geschlechter auf 75 weibliche (47\%) und 83 männliche (53\%) Patienten. Das durchschnittliche Alter in der PET/CT-Gruppe war mit $65 \pm 11$ Jahre signifikant niedriger als bei der CT-Gruppe mit $68 \pm 10$ Jahre $(p=0,008)$ ( $\bullet$ Abb. 1). Mit dem Stichtag 07.12.2010 waren 178/254 (70\%) Patienten der Protokollgruppe verstorben, von den 76 noch lebenden Patienten waren 39/96 (Überlebensrate: $41 \%$ ) in der PET/CT-Gruppe, $37 / 158$ (Überlebensrate: $23 \%$ ) in der CT-Gruppe $(\mathrm{p}=0,004)$.

Das mediane Überleben in der gesamten Protokollgruppe lag bei 246 Tagen (Spannweite: 217-275 Tage), entsprechend 8,2 Mo- naten, das der konventionell diagnostizierten Subgruppe lag bei 207 Tagen (Spannweite: 161 - 253 Tage), entsprechend 6,9 Monaten. Für die PET/CT-Gruppe ergab sich ein medianes Überleben von 338 Tagen (Spannweite: 247-429 Tage), entsprechend 11,3 Monaten $(\checkmark$ Tab.1 und $\bullet$ Abb. 2). Im Vergleich zeigte die PET/CTGruppe somit ein um 131 Tage (4,4 Monate) längeres Gesamtüberleben als die Patientengruppe mit konventioneller Diagnostik ( $\mathrm{p}=0,001$ Log-rank-Test).

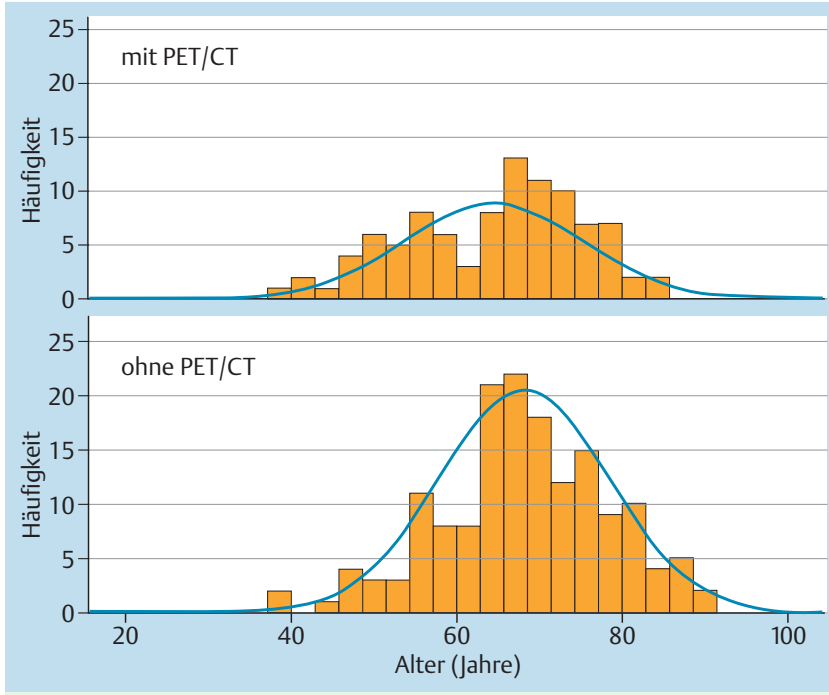

Abb. 1 Altersverteilung in Abhängigkeit von der Staging-Methode.

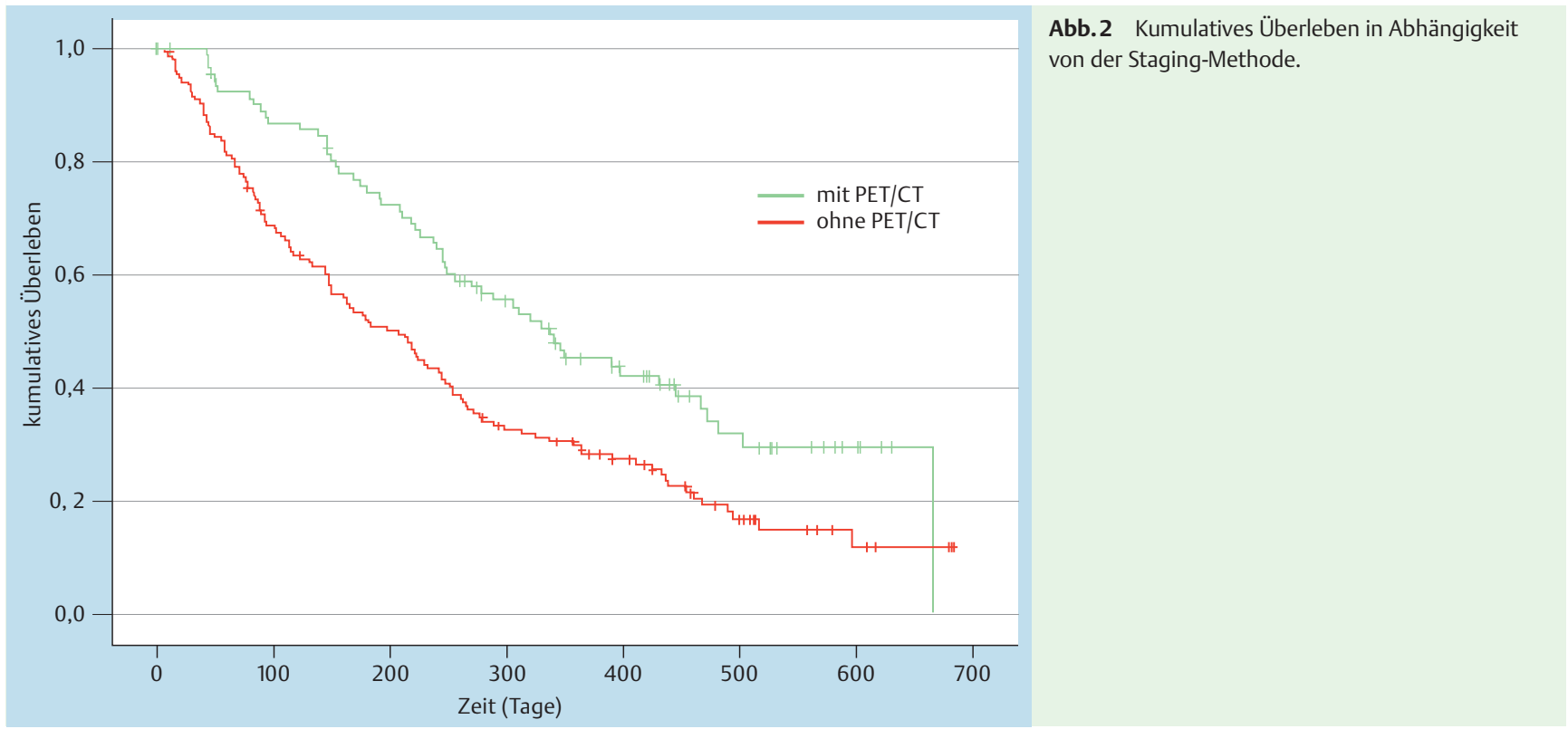

Tab. 1 Medianes und mittleres Überleben nach Kaplan-Meier, 95\% Konfidenzintervall angeben.

\begin{tabular}{|c|c|c|c|c|c|c|c|c|}
\hline \multirow[t]{3}{*}{ Gruppe } & \multicolumn{4}{|c|}{ Mittelwert } & \multicolumn{4}{|l|}{ Median } \\
\hline & \multirow[t]{2}{*}{ Wert } & \multirow[t]{2}{*}{ Std-Abw. ${ }^{1}$} & \multicolumn{2}{|c|}{ 95\% Konfidenzintervall } & \multirow[t]{2}{*}{ Wert } & \multirow[t]{2}{*}{ Std-Abw. ${ }^{1}$} & \multicolumn{2}{|c|}{$95 \%$ Konfidenzintervall } \\
\hline & & & untere Grenze & obere Grenze & & & untere Grenze & obere Grenze \\
\hline nur CT & 262,19 & 18,19 & 226,53 & 297,85 & 207,00 & 23,56 & 160,83 & 253,18 \\
\hline PET/CT & 374,91 & 24,59 & 326,70 & 423,11 & 338,00 & 46,61 & 246,65 & 429,35 \\
\hline Gesamt & 205,17 & 15,12 & 275,54 & 334,80 & 246,00 & 14,77 & 217,06 & 274,94 \\
\hline
\end{tabular}

${ }^{1}$ Standard-Abweichung. 

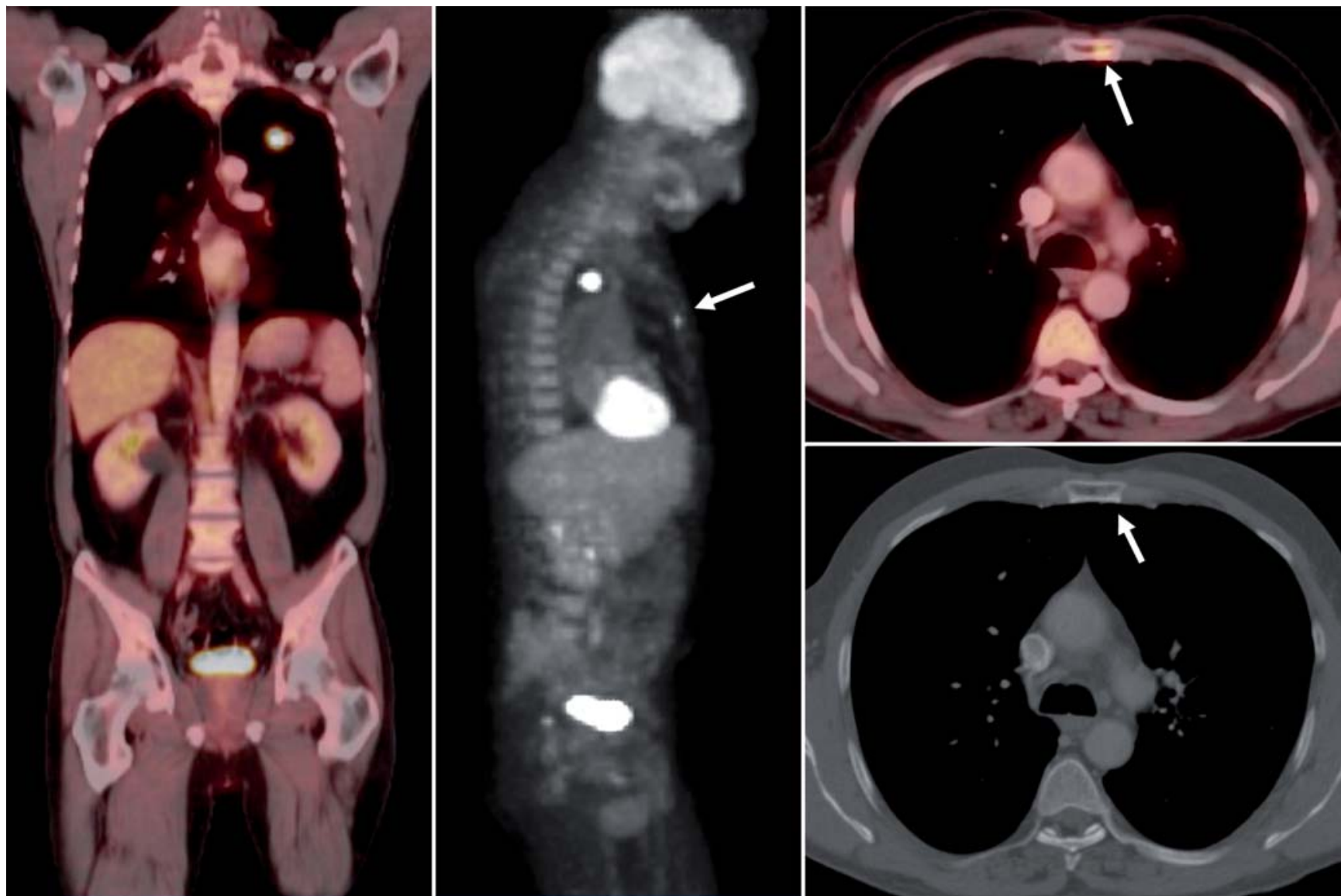

Abb.3 NSCLC linker Oberlappen. Im PET/CT unerwartete Detektion einer Sternummetastase (Pfeil; im Verlauf zytologisch gesichert).

\section{Diskussion \\ $\nabla$}

Studien, in denen diagnostische Verfahren, bezogen auf das Gesamtüberleben beim nicht-kleinzelligen Lungenkarzinom, verglichen wurden, sind rar, entsprechende Erkenntnisse für das palliative Stadium IV existieren derzeit nicht. Verlängerungen der Überlebenszeit innerhalb eines Tumorstadiums werden beim NSCLC in der Regel Veränderungen im therapeutischen Prozedere, zum Beispiel einer moderneren Chemotherapie, zugeschrieben.

So wurden 1982 noch Therapiestudien beim fortgeschrittenen NSCLC veröffentlicht, die mit Polychemotherapie das Gesamtüberleben von 8,5 Wochen auf 30,5 Wochen verlängert haben [13]. Moderne Therapiestudien erreichen zunehmend auch mediane Überlebenszeiten von deutlich mehr als 1 Jahr [14]. Ein Teil dieser Erfolge ist sicherlich einer strengen Selektion der Studienpatienten (keine Hirnfiliae, keine wesentlichen Nebenerkrankungen und/oder Laborauffälligkeiten etc.) geschuldet und somit nicht ohne weiteres auf ein unselektiertes Patientengut, zu dem der größere Teil unserer Patienten gehört, zu übertragen. Dennoch ist wohl insgesamt davon auszugehen, dass der überwiegende Teil der durchschnittlichen Überlebenszeitverlängerung auch beim fortgeschrittenen Lungenkarzinom auf eine verbesserte Therapie und eine intensivere Patientenversorgung einschließlich der Palliativmedizin zurückzuführen ist. Inwieweit jedoch die moderne Diagnostik einen unmittelbaren oder mittelbaren Einfluss auf das Überleben hat, ist unklar.

Im Vergleich von PET/CT und normalem CT zeigten die Patienten in der PET/CT-Gruppe in der aktuellen Untersuchung eine um 131
Tage längere mediane Überlebenszeit. Dieser deutliche Unterschied ist sicherlich zum Teil durch den besseren Performancestatus der Patienten in der PET/CT-Gruppe zu erklären, da diese Patienten ja zumeist für einen kurativen therapeutischen Ansatz (Operation oder zumindest Bestrahlung) vorgesehen waren und deshalb auch gewisse Mindestanforderungen bzgl. des Allgemeinzustands erfüllten. Eine weitere Erklärungsmöglichkeit ist aber, dass die FDG-PET/CT über die Detektion unerwarteter und ansonsten unerkannter Metastasen ( $\bullet$ Abb.3) das Stadium IV viel früher im individuellen Krankheitsverlauf diagnostiziert und die Patienten allein durch die frühere Diagnose „scheinbar“ länger leben. In einem theoretischen Lebenszeitmodell, wie in - Abb. 4 vereinfacht dargestellt, detektieren empfindlichere Nachweismethoden den möglichen Diagnosezeitpunkt eines Lungenkarzinoms (ED) sowie den Übergangszeitpunkt in ein metastasiertes Tumorstadium (M) früher und führen so zu therapieunabhängigen Lebenszeitverschiebungen innerhalb der Stadien. Darüber hinaus ermöglicht eine frühere Diagnose auch häufig eine intensivere und längere Therapie, was ebenfalls einen Einfluss auf das Überleben innerhalb des Stadiums IV haben kann. Bisher ist eine einzige Studie veröffentlicht, die der Frage nachgegangen ist, ob die Einführung des PET/CT in das Primärstaging beim NSCLC auch einen Einfluss auf das stadienbezogene Überleben hat. Diese Studie wurde mit primär operablen Patienten durchgeführt und der Einfluss des PET/CT auf das Gesamtüberleben der operierten Patienten untersucht [15]. Es wurde eine chirurgische Tumordatenbank retrospektiv ausgewertet und die Patienten mit einem präoperativen FDG-PET/CT denen mit konventionellem Staging gegenübergestellt. Während diese Studie 


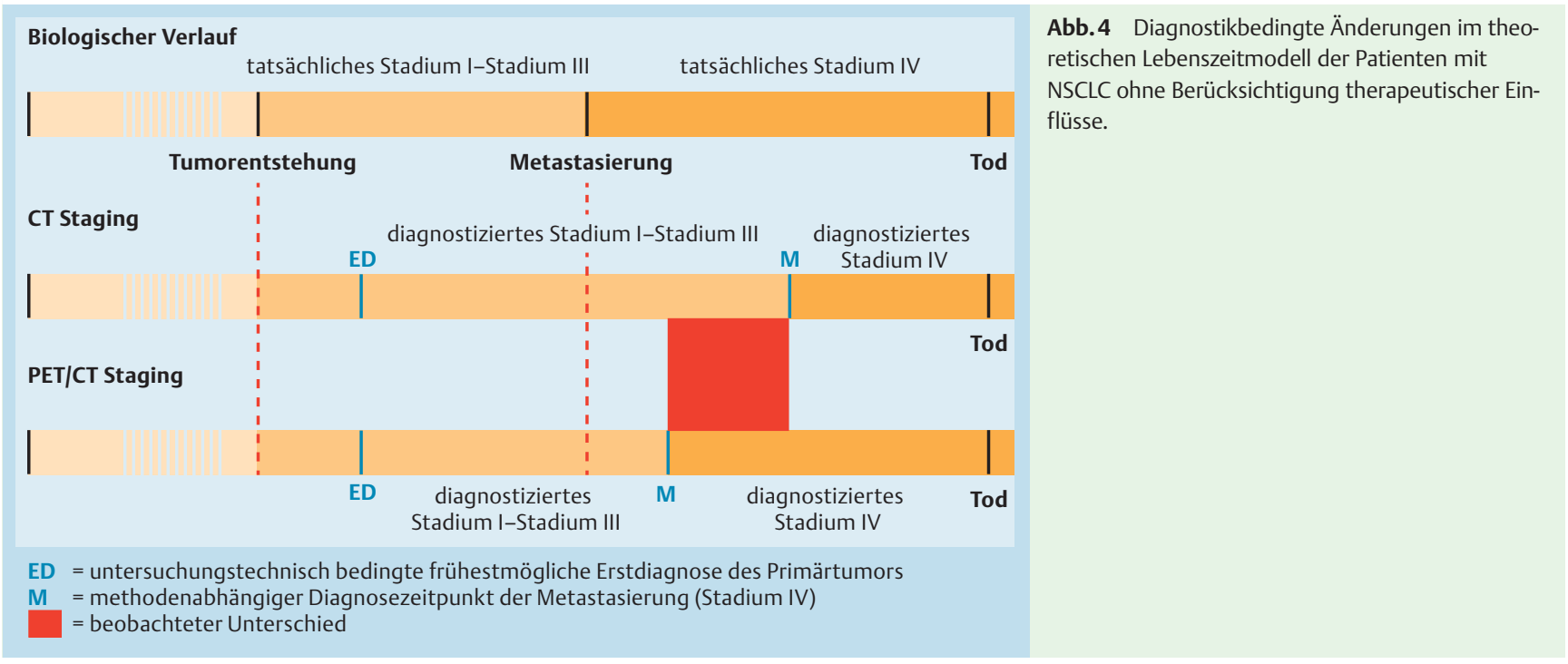

keinen Unterschied im Überleben für die operierten Stadien I und II nachweisen konnte, fand sich jedoch ein signifikanter Vorteil im Gesamtüberleben für das Stadium III zugunsten des präoperativen FDG-PET/CT [15]. Derartige Daten sind für das palliative Stadium IV bisher nicht veröffentlicht.

Wir wollten mit unserer Studie dieser Frage nachgehen und zunächst prüfen, ob die Patienten, die erst durch das FDG-PET/CT in ein Stadium IV umgruppiert werden, eine prognostisch günstigere Subgruppe darstellen. In Anlehnung an das 1985 beschriebene Will Rogers Phänomen [12] wäre dann, durch den zunehmenden Einsatz dieser Untersuchungstechnik, von einer relevanten Migration prognostisch günstigerer Patienten in das ungünstige Stadium IV auszugehen und eine konsekutive Verlängerung des Gesamtüberlebens in diesem Stadium zu erwarten. Dies wäre dann einzig die Folge einer verbesserten Diagnostik und therapieunabhängig.

Für die Studie wurde ein retrospektives Fall-Kontroll-Design verwendet, da bei der hohen Sensitivität ein prospektives Design nur schwer umsetzbar ist. Deshalb sind einige methodenimmanente Einschränkungen zu berücksichtigen. Das PET/CT wurde im Jahr 2009 vornehmlich präoperativ zum Ausschluss von Fernmetastasen und bzgl. des Lymphknotenstagings eingesetzt. Patienten mit bereits bekannten und/oder symptomatischen Metastasen (Schmerzen, neurologische Symptomatik etc.) erhielten in der Regel kein FDG-PET/CT. Auch inoperable Patienten wurden mittels FDG-PET/CT zum Ausschluss von Fernmetastasen untersucht, wenn eine Bestrahlung in kurativer Intention erwogen wurde. Der Anteil an Patienten, die durch unerwartete Metastasen in ein Stadium IV hochgestuft wurden, ist somit in unserem Kollektiv wahrscheinlich überrepräsentiert. Darüber hinaus ist beim Gesamtüberleben immer von einem multikausalen Einfluss auszugehen, insbesondere wenn Einflüsse am Beginn der Diagnose beschrieben werden wollen. Alle Therapieansätze in der ersten und mit Einschränkung auch in der zweiten Linie sind in diesem Zeitraum als überwiegend homogen zu bezeichnen, sodass ein gerichteter Effekt der Therapie in dieser Studie unwahrscheinlich erscheint.

Mit 246 Tagen (35,1 Wochen bzw. 8,2 Monate) ist das mediane Gesamtüberleben aller Patienten mit einem in 2009 an unserer Klinik neudiagnostizierten, metastasierten NSCLC für ein unse- lektiertes Patientengut repräsentativ. Verglichen mit den Überlebensdaten, die von der IASLC noch 2007 für das Stadium IV veröffentlicht wurden, ist das mediane Überleben der konventionell stadiierten Patienten mit 207 Tagen (29,6 Wochen, 6,9 Monate) vergleichbar [2]. Das mediane Überleben der PET/CT-Gruppe dagegen liegt mit 338 Tagen (48,3 Wochen, 11,3 Monaten) im Bereich moderner Therapiestudien, die in aller Regel ein hoch selektiertes Patientenkollektiv untersuchen [16-18].

Unsere Untersuchung zeigt erstmals, dass die moderne Bildgebung durch FDG-PET/CT therapieunabhängig durch eine frühere Diagnose höherer Tumorstadien zu einer Verlängerung des Gesamtüberlebens im metastasierten Stadium beim NSCLC beitragen kann. Da es sich nicht um eine bzgl. der Patientencharakteristika gepaarte oder gar prospektiv randomisierte Studie handelt, ist die errechnete Lebenszeitverlängerung zwar nur eingeschränkt valide, der gezeigte Effekt der Stagingmethode auf das Überleben im Stadium IV aufgrund seines Ausmaßes aber auch nicht zu negieren.

Bei der Interpretation von möglichen Therapieerfolgen im Stadium IV NSCLC, insbesondere im Rahmen von Therapiestudien, sollte zukünftig auch die angewandte Diagnostikmethode berücksichtigt werden, da der gezeigte Effekt deutlich über den aktuell geforderten Therapieeffekten liegt. Vergleiche mit historischen Kollektiven sollten immer auch diagnostikbedingte Einflüsse berücksichtigen.

\section{Danksagung}

Wir danken Herrn Thorsten Bergmann für die Mitarbeit am Manuskript.

\section{Interessenkonflikt}

$\nabla$

Die Autoren geben an, dass kein Interessenkonflikt besteht. 


\section{Literatur}

1 Statistisches Bundesamt Deutschland. Lungenkrebs ist die vierthäufigste Todesursache. Wiesbaden: 2007. www.destatis.de/jetspeed/ portal/cms/Sites/destatis/Internet/DE/Presse/pm/2007/05/

PD07_217_232.psml (Stand: 18.11.2007)

2 Groome PA, Bolejack V, Crowley JJ et al. The IASLC Lung Cancer Staging Project: Validation of the Proposals for Revision of the T, N and M Descriptors and Consequent Stage Groupings in the Forthcoming (Seventh) Edition of the TNM Classification of Malignant Tumours. J. Thorac Oncol 2007; 2: 694-705

3 Verhagen AF, Bootsma GP, Tjan-Heijnen VC et al. FDG-PET in staging lung cancer: how does it change the algorithm? Lung Cancer 2004; 44: $175-181$

4 Beyer T, Townsend DW, Brun T et al. A combined PET/CT scanner for clinical oncology. J. Nucl Med 2000; 41: 1369-1379

5 Kluetz PG, Meltzer CC, Villemagne VL et al. Combined PET/CT imaging in oncology. Impact on patient management. Clin Positron Imaging 2000; 3: $223-230$

6 Ung YC, Maziak DE, Vanderson JA et al. 18-FDG-PET in the diagnosis and staging of lung cancer: A systematic review. J Natl Cancer Inst 2007; 99: $1753-1767$

7 Hellwig D, Ukena D, Paulsen $F$ et al. Metaanalyse zum Stellenwert der Positronen-Emissions-Tomographie mit F-18-Fluorodesoxyglukose (FDG-PET) bei Lungentumoren. Pneumologie 2001; 55: 367-377

8 Goeckenjan G, Sitter H, Thomas $M$ et al. Prävention, Diagnostik, Therapie und Nachsorge des Lungenkarzinoms. Interdisziplinäre S3-Leitlinie der Deutschen Gesellschaft für Pneumologie und Beatmungsmedizin und der Deutschen Krebsgesellschaft. Pneumologie 2010; 64 (Suppl. 02): e1 - e164

9 Fischer B, Lassen U, Mortensen J et al. Preoperative Staging of Lung Cancer with Combined PET-CT. N Engl J Med 2009; 361: 32 - 39

10 Herder GJM, Kramer H, Hoekstra OS et al. Traditional Versus Up-Front [18F] Fluorodeoxyglucose-Positron Emission Tomography Staging of Non-Small-Cell Lung Cancer: A Dutch Cooperative Randomized Study. J Clin Oncol 2006; 24: $1800-1806$
11 Lee DH, Kim SK, Lee HY et al. Early Prediction of Response to First-Line Therapy Using Integrated 18F-FDG PET/CT for Patients with Advanced/ Metastatic Non-small Cell Lung Cancer. J Thorac Oncol 2009; 4: 816 821

12 Feinstein AR, Sosin DM, Wells CK. The Will Rogers Phenomenon. Stage Migration and New Diagnostic Techniques as a Source of Misleading Statistics for Survival in Cancer. N Engl J Med 1985; 312: 1604-1608

13 Cormier Y, Bergeron D, La Forge J et al. Benefits of Polychemotherapy in advanced non-small-cell bronchogenic carcinoma. Cancer 1982; 50: $845-849$

14 Reck M, von Pawel J, Zatloukal $P$ et al. Overall survival with cisplatingemcitabine and bevacizumab or placebo as first-line therapy for nonsquamous non-small-cell lung cancer: results from a randomised phase III trial (AVAiL). Ann Oncol 2010; 21: 1804-1809

15 Fontaine E, McShane J, Carr $M$ et al. Does positron emission tomography scanning improve survival in patients undergoing potentially curative lung resections for non-small-cell lung cancer? Eur J Cardiothorac Surg 2008; 34: 892-897

16 Butts CA, Bodkin D, Middleman EL et al. Randomized phase II study of gemcitabine plus cisplatin or carboplatin [corrected], with or without cetuximab, as first-line therapy for patients with advanced or metastatic non small-cell lung cancer. J Clin Oncol 2007; 25: 5777- 5784 Erratum in: J Clin Oncol 2008; 26: 3295.

17 Treat J, Scagliotti GV, Peng G et al. Comparison of pemetrexed plus cisplatin with other first-line doublets in advanced non-small cell lung cancer (NSCLC): A combined analysis of three phase 3 trials. Lung Cancer 2011 (in press)

18 Scagliotti GV, Parikh P, von Pawel J et al. Phase III Study Comparing Cisplatin Plus Gemcitabine With Cisplatin Plus Pemetrexed in Chemotherapy-Naïve Patients With Advanced-Stage Non-Small-Cell Lung Cancer. J Clin Oncol 2008; 26: 3543-3551 